\title{
RESEARCH
}

Open Access

\section{Modelling of thermomechanical behaviour of fibrous polymeric composite materials subject to relaxation transition in the matrix}

Valeriy Pavlovich Matveenko', Nikolay Alexandrovich Trufanov ${ }^{2 *}$, Oleg Yurievich Smetannikov², Igor Nikolaevich Shardakov ${ }^{1}$ and Igor Nikolaevich Vasserman ${ }^{1}$

\begin{abstract}
Background: Fiber-reinforced polymer composite materials are widely used in different branches of industry due to their distinctive features such as high specific strength and stiffness and due to as considerable opportunity to formulate materials with controllable variation of properties in response to the action of external factors (smartmaterials). A distinguishing feature of products made of composite materials is that the processes of product and material fabrication are inseparable. Therefore the estimation of composite properties based on the composite architecture and properties of the reinforcing fibers and matrix is a very actual task.

Methods: The model of polymer behavior at glass transition recently developed by the authors was generalized to the case of fiber-reinforced polymer matrix composites using two approaches: one is base on the concept of free specific energy, the other - on the growth of matrix stiffness. For homogeneous materials these two approaches are of equal worth, whereas for composite materials they give different results under deformation in the transverse direction. The stiffness growth approach is more accurate, but is very expensive computationally and, is highly sensitive to the experimental data errors.

Results: Using the finite element method and averaging technique the thermoelastic constants of composites containing different types of fibers in the glassy and high-elastic states were calculated based on the fiber and matrix properties. Softening of the matrix has an insignificant effect on the longitudinal modulus of a composite but leads to a considerable decrease of the transverse and shear moduli. The coefficient of thermal expansion in the transverse direction is much higher than the coefficient of thermal expansion in the longitudinal direction, especially when the composite is in the high-elastic state.

Conclusion: The model of polymer behavior at glass transition recently developed by the authors can be generalized to the case of fiber-reinforced polymer matrix composites. The thermoelastic constants of composites containing different types of fibers can be calculated from the fiber and matrix properties using the finite element method and averaging technique.
\end{abstract}

Keywords: Fiber-reinforced composites, Glass transition, Glass and high-elastic state of polymer

\footnotetext{
*Correspondence: nat@pstu.ru

${ }^{2}$ Perm National Research Polytechnic University, Perm, Russian Federation

Full list of author information is available at the end of the article
} 


\section{Background}

Fiber-reinforced polymer composite materials are widely used in different branches of industry due to their distinctive features Among technical characteristics of considerable commercial importance are high specific strength and stiffness, low specific density, high fracture toughness and fatigue strength, as well as considerable opportunity to formulate materials with controllable variation of properties in response to the action of external factors (smart-materials).

The materials under consideration consist of fibrous filling material, which when combined with a polymer binding material, forms a fiber-reinforced polymer matrix composite. As a rule, reinforcing fibers have high longitudinal stiffness and tensile strength, while a composite matrix has high toughness, which provides superior mechanical characteristics of composites. The structure of these materials is responsible to the anisotropy of their physico-mechanical properties.

A distinguishing feature of products made of composite materials is that the processes of product and material fabrication are inseparable. It can be said with assurance that each product is unique in showing properties that may be different in different its parts. It means that physico-mechanical properties of the product cannot be estimated by a standard approach from the test data of specimens made of the same material. Therefore at present, the problem of current concern is the estimation of composite properties based on the composite architecture and properties of the reinforcing fibers and matrix.

The process of composite formation proceeds in a certain temperature range. The temperature regimes arising in this interval are accompanied by relaxation transitions to glassy and rubber-like states in the polymer matrix. Organic plastic materials also demonstrate a strong dependence of fiber mechanical properties on temperature. All these thermomechanical processes give rise to residual/technological stresses.

Predictive modeling of the composite behavior based on the properties of its components is the objective of many studies (Christensen 1979; Hill 1964a, b; 1965a, b; 1966; Malmeister et al. 1980; Pobedrya 1964; Sokolkin $\mathrm{Yu}$ and Tashkinov 1984; Vildeman et al. 1997). Thus, works (Christensen 1979; Hill 1964a, b; 1965a, b; 1966; Malmeister et al. 1980; Vildeman et al. 1997) are devoted to modeling the thermomechanical behavior of fiberreinforced composites. The focus of authors' attention in these studies are such problems as estimation of elastic moduli of composite materials based on the properties of their components (Christensen 1979; Malmeister et al. 1980; Pobedrya 1964; Sokolkin Yu and Tashkinov 1984, Dinzart and Lipiński 2010, Dupaix and Boyce 2006, Ge et al. 2012), modeling of nonlinear and transient processes in a composite material (Vildeman et al. 1997, Bugakov 1989), damage accumulation and fracture of composites (Sokolkin Yu and Tashkinov 1984; Vildeman et al. 1997, Bugakov 1989, Christensen 1979). However, the above studies are concerned mainly with the processes of deformation and fracture in finished composites.

On the other hand, there are a lot of papers (Askadskiy 1973; Bartenev and Zelenev 1976; Begishev et al. 1997; Brader et al. 2009; Buckley and Jones 1995; Bugakov 1989; Dinzart and Lipiński 2010; Dupaix and Boyce 2006; Henann and Anand 2008; Liu et al. 2006; Lustig et al. 1996; Nguen et al. 2008; Qi et al. 2008; Scalet et al. 2015; Shardakov and Golotina 2009; Sollich 1998; Tervoort et al. 1996; Xiao et al. 2013) (including the works by the present authors (Matveenko et al. 2012; 2015), which investigate the thermomechanical behavior of homogeneous polymers (not composites) at glass transition/softening temperatures. The effect of shape memory, which is a frequent consequence of thermorelaxation transitions, is investigated in (Dinzart and Lipiński 2010; Liu et al. 2006; Lustig et al. 1996; Nguen et al. 2008; Qi et al. 2008; Scalet et al. 2015; Shardakov and Golotina 2009; Xiao et al. 2013).

Simulation of thermorelaxation transitions in composite materials is a less explored area, which at present is being studied intensively. Most of recently published works on this subject are concerned with composite smart materials exhibiting the shape memory effect (Ge et al. 2012; 2014; Mulina and Sawant 2009; Tan et al. 2014, Srivastava et al. 2010a, b, Tervoort et al. 1996), in which the relaxation and phase transitions can occur both in the matrix (Ge et al. 2014; Mulina and Sawant 2009; Tan et al. 2014) and in the reinforcing fibers (Ge et al. 2012).

In this paper, we generalize a thermomechanical model of composite material undergoing glass transition developed early by the authors in (Matveenko et al. 2012; 2015) to the case of fiber-reinforced composite materials with polymeric matrix, which due to variation of temperature can undergo transition from the high-elastic to the glassy state and back. The relations constructed in these papers are based on the specific free energy of the composite, which is used as a scalar measure of the state of the material undergoing glass transition. We introduce a technique for determining the equation parameters in terms of the effective properties of the composite. We also develop a more general model, in which glass transition is considered as the process of enhancement of the matrix stiffness. We compare the two models to show their advantages and drawbacks. A numerical technique has been proposed to determine the effective material constants and functions for unidirectional fiber-reinforced composites with epoxy matrix undergoing glass transition due to 
temperature variation. The investigation has been performed taking no account of viscous elasticity.

\section{Methods \\ Polymer matrix}

The polymer matrix consists of very long, linear, branched or cross-linked macromolecules, which comprise a large number of repeatable chains. The structure of the macromolecule is specified by configuration and conformation. Configuration is the mutual arrangement of atoms in the molecule, which cannot be changed without breaking the existing chemical bonds. Conformation is the relative spatial arrangement of atoms in the molecule, which can vary under the action of thermal motion by rotation around single carbon-carbon bonds. Depending on the structure the polymers can be classified as thermoplastics and reactoplastics. Thermoplastics have a linear or branched structure and can transform from a plastic to a solid state and change back. Reactoplastics possess a spatial cross-linked structure, in which the neighboring chains are linked by chemical covalent bonds, the strength of which is as high as the strength of the bonds in the polymer chains. Polymers of this group are unable to pass into a reversible plastic state. They can only decompose chemically when heated to certain temperatures. The molecules of polymers can form supermolecular structures, one being knows as a fluctuation network. Physically, this implies that some segments of molecules in microvolumes form the short-range order structures with higher density and more intensive intermolecular interaction (fluctuation network nodes). Subject to thermal or mechanical loads these structures may decay in some places and emerge in other places.

The above mentioned features of the molecular structure specifies the behavior of solid polymers, which depending on temperature and mechanical loads can exist in two states - glassy and high-elastic. At low temperatures the polymer behaves as glass. Under the action of applied stresses the conformational motion of polymer chains does not occur, which means that the shape of macromolecules does not change. Deformation in this state is small and is related to changes in the interatomic distances and bond angles and also to a change in the average intermolecular space.

Beginning with some temperature the deformability of a polymer increases causing its transition into highelastic state. In this state the strain can be as high as a few hundred percent and is associated with the conformational movements of macromolecules, the failure of fluctuation network nodes in some places and their formation in other less strained places. It should be noted that a transition from the glassy to the highelastic state is not an instantaneous process. It occurs within a certain temperature range, which suggests that there exists some transitional region $T_{g 2} \leq T \leq T_{g 1}$.
At temperature lower than the glass transition temperature and high level of stresses the polymer undergoes forced high-elastic-deformation, which involves unfreezing of segmental mobility of macromolecules.

To describe the thermomechanical behavior of polymers undergoing glass transition we use the model developed by the authors in (Matveenko et al. 2012). The model has been elaborated using the following hypothesis:

1) The characteristic times of external actions are much shorter than the relaxation times of a polymer in the glassy state.

2) Characteristic times of external actions are far beyond the limits of the relaxation spectrum of a polymer in the high-elastic state.

In this case, outside the glass transition temperature range the polymer behaves as an elastic material. The behavior of the polymer matrix in the glass transition temperature range at decreasing temperature is specified by increase in the mechanical stiffness of the polymer due to a decrease in the segmental mobility of molecules and a growth of the energy of intermolecular interaction. At the phenomenological level this looks like a superposition of additional elastic bonds onto the original polymer network in the high-elastic state. Note that each of these bonds at the time of its formation is assumed to be nondeformed.

The degree of completeness of glass transition process of a polymer-matrix composite is described by the quantity $N \in[0,1]$ called degree of vitrification. Here 0 refers to a polymer in the high-elastic state, 1 - to a polymer in the glassy state. A change in the degree of vitrification at time $t_{j}$ by $\Delta N_{j}$ is accompanied by an increase of the free energy of additional bonds in the vitrified material by the amount $F_{2}\left(\varepsilon-\varepsilon_{j}\right) \Delta N_{j}$. Here $F_{2}$ is the free energy of additional bonds in the fully vitrified polymer and $\varepsilon_{j}$ is the strain at time $t_{j}$. Thus, for a polymer in the state corresponding to the degree of vitrification (conversion) $N(t)$ the expression for free energy is

$$
F(\varepsilon(t))=F_{1}(\varepsilon(t))+\int_{0}^{N(t)} F_{2}(\varepsilon(t)-\varepsilon(\tau)) d N(\tau),
$$

where $F_{1}$ is the free energy of the material in the highelastic state.

For the isotropic polymer the expression for free energy in the high-elastic state and the energy of bonds formed in the glass transition temperature range is given as

$$
F_{i}=\frac{1}{2}\left(B_{i}+\frac{4}{3} G_{i}\right) I_{1}^{2}-2 G_{i} I_{2}-3 B_{i} I_{1} \varepsilon_{T}, \quad i=1,2,
$$

where $I_{1}=\theta=\varepsilon_{k k}, I_{2}=0.5\left(\varepsilon_{m m} \varepsilon_{n n}-\varepsilon_{m n} \varepsilon_{m n}\right)$ are the first 
and the second invariants of the strain tensor, $G_{2}=G_{g}$ $G_{1} ; B_{2}=B_{g}-B_{1} ; G_{1}, B_{1}$ are the shear and bulk moduli in the high-elastic state, $G_{g}, B_{g}$ are the shear and bulk moduli in the glassy state, $\varepsilon_{T}=\int_{T_{H}}^{T(t)} \alpha(T) d T$ is the temperature deformation.

The physical relations are written on the basis that

$$
\sigma_{i j}=\frac{1}{2}\left(\frac{\partial F}{\partial \varepsilon_{i j}}+\frac{\partial F}{\partial \varepsilon_{j i}}\right),
$$

which leads to

$$
\begin{gathered}
s_{i j}(t)=2 G_{1} e_{i j}(t)+2 G_{2} \int_{T_{H}}^{T(t)}\left[e_{i j}(t)-e_{i j}(\tau)\right] d N(T(\tau)) \\
\sigma(t)=B_{1}\left[\theta(t)-3 \varepsilon_{T}(t)\right]+B_{2} \int_{T_{H}}^{T(t)}\left\{\left[\theta(t)-3 \varepsilon_{T}(t)\right]\right. \\
\left.-\left[\theta(\tau)-3 \varepsilon_{T}(\tau)\right]\right\} d N(T(\tau))
\end{gathered}
$$

where

$$
e_{i j}=\varepsilon_{i j}-\frac{1}{3} \theta \delta_{i j} ; \quad s_{i j}=\sigma_{i j}-\sigma \delta_{i j} ; \quad \sigma=\frac{1}{3} \sigma_{k k} .
$$

For a detailed description of the glass transition process it is necessary to evaluate the dependence of the degree of conversion on time $N(T)$. As opposed to the process of crystallization, the process of structural glass transition is not accompanied by generation or absorption of heat. It means that a certain value of temperature (varying with a constant rate) is associated with a certain value of the conversion degree $N(T)$. Typical $N(T)$ curves are displayed in Fig. 1. Such relationships are well described by the probability density functions widely used in the mathematical statistics. The reason why the preference is given to these function is that in compliance with the molecular-kinetic theory (Word 1975) a decrease in the segmental mobility of polymers undergoing glass transition is a stochastic process.

Particularly, in the case of symmetric distribution of function $N(T)$ with respect to the glass transition temperature there is good reason to use an expression for the Gaussian probability density function. In this case, with reference to the rate of variation of conversion degree we can write

$$
\frac{d N_{G}}{d T}=-\frac{1}{\sqrt{2 \pi} \gamma} \exp \left[-\frac{1}{2}\left(\frac{T-T_{g}(\dot{T})}{\gamma_{G}}\right)\right]^{2},
$$

where index «G» denotes the use of the Gaussian distribution law. Dispersion $\gamma$ is determined in terms of the breadth

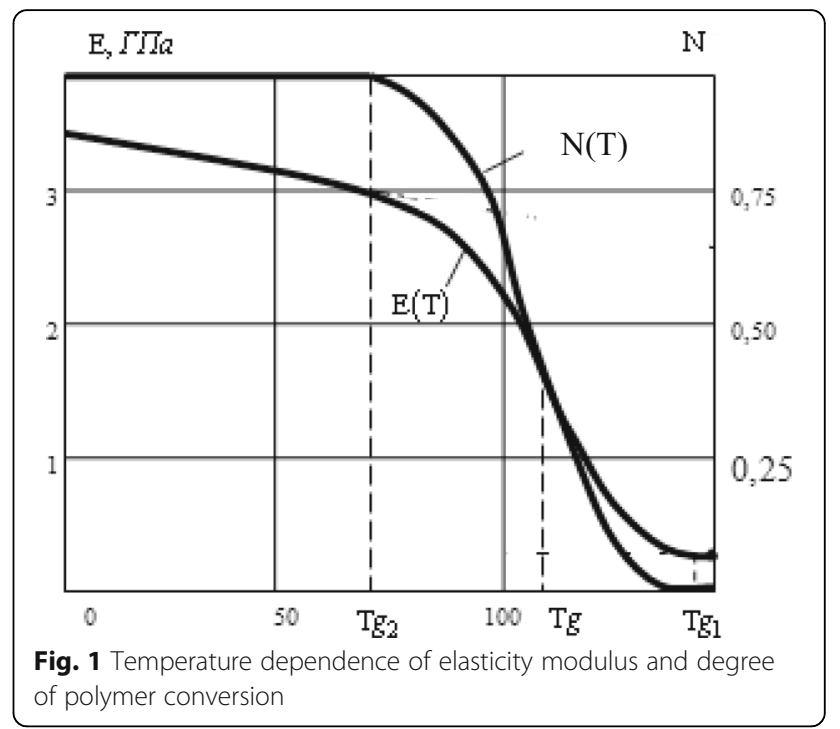

of the glass transition interval using formula $\gamma_{G} \cong\left(T_{g 1}-\right.$ $\left.T_{g_{2}}\right) / 6$, and the glass transition temperature $T_{g}$ is taken as a mathematical expectation. In the general case $T_{g}$ should be considered dependent on the rate of temperature variation $\dot{T}$, which is a well-known fact supported by experiments (Word 1975, Xiao et al. 2013). The minus sign suggests an increase in the degree of conversion $N(T)$ on cooling. The degree of conversion corresponding to temperature $T(t)$ will be determined by integrating the expression for the rate of polymer conversion

$$
N_{G}(T(t))=-\frac{1}{\sqrt{2 \pi} \gamma} \int_{T_{H}}^{T(t)} \exp \left[-\frac{1}{2}\left(\frac{T(\tau)-T_{g}(\dot{T})}{\gamma_{G}}\right)^{2}\right] d T(\tau) .
$$

There are some other more convenient formulas, which describe the kinetics of glass transition based on the analytical relationships between the rate of conversion, temperature and rate of temperature variation. One of such models is the Laplace distribution, which can be applied in the case of symmetric dependence of the conversion degree on temperature.

$$
N_{L}(T, \dot{T})= \begin{cases}1-0,5 e^{\frac{\left(T-T_{g}(\dot{T})\right)}{\gamma_{L}}}, & T<T_{g} \\ 0,5 e^{\frac{\left(T-T_{g}(\dot{T})\right)}{\gamma_{L}}}, & T \geq T_{g}\end{cases}
$$

where $\gamma_{L}$ is the parameter determining the width of the glass transition temperature range.

If the distribution $N(T)$ shows the pronounced asymmetry about some conventional central point in the glass transition temperature range, one should use the asymmetrical laws of probability distribution, for example the Weibull distribution law 


$$
N_{W}(T, \dot{T})=\left\{\begin{array}{cc}
1, & T<T_{g W} ; \\
e^{-\lambda\left(T-T_{g W}(\dot{T})\right)^{\beta}}, & T \geq T_{g W} .
\end{array}\right.
$$

\section{Reinforcing fibers}

Reinforcing fibers are elastic isotropic (fiberglass), elastic anisotropic (boron fiber, carbon fiber) and temperature dependent elastic anisotropic (organic fiber) materials. The constitutive relations for these materials can be written in the general form as

$$
\sigma_{i j}=C_{i j k l}^{a}(T)\left[\varepsilon_{k l}-\varepsilon_{T k l}^{a}(T)\right] .
$$

\section{Constitutive relations for fiber reinforced composites}

The model developed by the authors in (Matveenko et al. 2012) can be generalized to the description of fiber reinforced composites undergoing glass transition. As in the case of isotropic material, two approaches can be applied. The former relies on the concept of free energy and the latter - on the concept stiffness enhancement. In the isotropic case, these two approaches yield identical results. In the anisotropic case, the results are found to be different.

Let us consider the first approach, in which $F_{1}$ denotes the specific free energy of the composite with highly elastic binding medium, and $F_{2}$ denotes the specific free energy of additional bonds in the completely vitrified composite. We assume that the degree of conversion $N(T)$ is described by the formula identical to that of the binding material. Then the expression for the specific free energy of composite material can be written as

$$
F(\hat{\varepsilon}(t))=F_{1}(\hat{\varepsilon}(t))+\int_{0}^{N(t)} F_{2}(\hat{\varepsilon}(t)-\hat{\varepsilon}(\tau)) d N(\tau),
$$

where

$$
\begin{aligned}
F_{1}(\varepsilon) & =\frac{1}{2} C_{i j k l}^{(1)} \varepsilon_{i j} \varepsilon_{k l}-C_{i j k l}^{(1)} \varepsilon_{i j} \varepsilon_{k l}^{T 1}-S_{1} T ; \\
F_{2}(\varepsilon) & =\frac{1}{2} C_{i j k l}^{(2)} \varepsilon_{i j} \varepsilon_{k l}-C_{i j k l}^{(2)} \varepsilon_{i j} \varepsilon_{k l}^{T 2}-S_{2} T ; \\
\varepsilon_{k l^{T 1}} & =\int_{T_{H}}^{T} \alpha_{k l}^{(1)}(T) d T ; \varepsilon_{k l}^{T 2}=\int_{T_{H}}^{T} \alpha_{k l}^{(2)}(T) d T
\end{aligned}
$$

$C_{i j k l}^{(1)}, \quad \alpha_{k l}^{(1)}$ are the components of the effective stiffness tensors and coefficients of linear temperature expansion of the composite at $T \geq T_{g 1} ; C_{i j k l}^{(2)}, \quad \alpha_{k l}^{(2)}$ are the same quantities for bonds formed in the process of vitrification of the matrix. The procedure of evaluating $C_{i j k l}^{(2)}$, $\alpha_{k l}^{(2)}$ in terms of the composite properties in the highelastic $C_{i j k l}^{(1)}, \alpha_{k l}^{(1)}$ and glassy $C_{i j k l}^{g}, \quad \alpha_{k l}^{g}$ states is given below.

After submitting (11) into (10) we get

$$
\begin{aligned}
\sigma_{i j}(t)= & \frac{1}{2}\left(\frac{\partial F}{\partial \varepsilon_{i j}}+\frac{\partial F}{\partial \varepsilon_{i j}}\right) \\
= & C_{i j k l}^{(1)}(T) \varepsilon_{k l}(t)+C_{i j k l}^{(2)}(T) \int_{T_{H}}^{T(t)}\left[\varepsilon_{k l}(t)-\varepsilon_{k l}(\tau)\right] d N(T(t)) \\
& -C_{i j k l}^{(1)}(T) \varepsilon_{k l}^{T 1}(t)-C_{i j k l}^{(2)}(T) N(T) \varepsilon_{k l}^{T 2}(t) \\
& +C_{i j k l}^{(2)}(T) \int_{T_{H}}^{T(t)} \varepsilon_{k l}^{T 2}(\tau) d N(T(\tau))
\end{aligned}
$$

To determine $C_{i j k l}^{(2)}, \quad \alpha_{k l}^{(2)}$ we consider the behavior of the composite in the glassy state $\left(T>T_{g 2}\right)$. One should bear in mind that the vitrification degree $N=1$ does not change with temperature, so that the integrals in the right-hand sides of (12) turn into constant quantities. Let at a fixed temperature $T$ the material deform, which implies that the strain tensor components gain the increment $\Delta \varepsilon_{k e}$. In this case the stress varies as

$$
\Delta \sigma_{i j}=\left(C_{i j k l}^{(1)}(T)+C_{i j k l}^{(2)}(T)\right) \Delta \varepsilon_{k l}
$$

Since the composite according to the problem formulation is considered as fully cured, the coefficient of proportionality between $\Delta \sigma_{i j}$ and $\Delta \varepsilon_{k l}$ is the component of the elasticity tensor of the material in the glassy state $C_{i j k l}^{(2)}$. Then it follows that

$$
C_{i j k l}^{(2)}=C_{i j k l}^{g}-C_{i j k l}^{(1)}
$$

We assume further that the material maintaining the same temperature is heated by $\Delta T$. Considering the material as being unstrained, we obtain from (12) the expression for stress increments

$$
\Delta \sigma_{i j}^{T}=-C_{i j k l}^{(1)}(T)-C_{i j k l}^{(2)}(T) \Delta \varepsilon_{k l}^{T 2}
$$

where $\Delta \varepsilon_{k l}^{T i} \equiv \varepsilon_{k l}^{T i}(\Delta T)$. Then we define the same quantity in terms of the effective characteristics of the composite in the glassy state

$$
\Delta \sigma_{i j}^{T}=-C_{i j k l}^{g}(T) \Delta \varepsilon_{k l}^{T g}
$$

where $\Delta \varepsilon_{k l}^{T g}=\alpha_{k l}^{g}(T) \Delta T$. Equating the right-hand sides of (14), (15), we obtain in the general case the system of six equations for the unknown independent components of tensor $\Delta \varepsilon_{k l}^{T g}$. For transversally isotropic and orthotropic materials the number of independent components of the strain tensor reduces to three. 


$$
\sum_{j=1}^{3} \Delta \varepsilon_{i i}^{T 2} C_{j j i i}^{(2)}(T)=\sum_{j=1}^{3}\left(\Delta \varepsilon_{i i}^{T g} C_{j j i i}^{g}(T)-\Delta \varepsilon_{i i}^{T 1} C_{j j i i}^{(1)}(T)\right)
$$

Solving the system of three linear Eq. (16), we obtain the sought values of $\Delta \varepsilon_{i i}^{T 2}$. If $\Delta T=1$, then $\alpha_{i i}^{(2)}=\Delta \varepsilon_{i i}^{T 2}$, and the free deformation $\varepsilon_{i i}^{T 2}$ can be determined using the formula

$$
\varepsilon_{i i}^{T 2}=\int_{T_{H}}^{T(t)} \alpha_{i i}^{(2)}(T) d T
$$

For approximate calculations it is allowable to take $\alpha_{11^{(2)}}=\alpha_{11}^{g}, \quad \alpha_{22}^{(2)}=\alpha_{33}^{(2)}=0,5\left(\alpha_{22}^{(1)}+\alpha_{22}^{g}\right)$.

The above technique of constructing the physical relations for composite materials with a vitrifying matrix (12) is based on the concept of free energy. The value of the free energy is invariant to the choice of coordinate system and does not take into account the anisotropy of the contribution of the vitrification degree to polymer stiffness. The isotropic polymers are lost to such effect, whereas composite materials characterized by a considerable directional variation of properties are rather sensitive to the conversion degree. The second stiffness-based approach, which like the free energy approach, relies on the same physical and phenomenological considerations, allows us to eliminate this drawback.

Let us consider the process of nonisothermal deformation of composite material, which at the initial moment of time is heated to the temperature above the glass transition interval. Under these conditions the composite behaves itself as an elastic medium with the properties $\hat{C}(0, T), \hat{\alpha}(0, T)$. Here $\hat{C}-$ is the fourth-order tensor of stiffness properties of the medium, $\hat{\alpha}$ is the second-order tenor of the effective linear coefficients of thermal expansion. For both tensors the temperature is taken to be the second argument and the degree of conversion - the first argument. Let at time $t_{1}$, corresponding to entering into the glass transition interval $\left(T\left(t_{1}\right) \leq T_{g 1}\right)$, a decrease in temperature by $\Delta T_{1}$ gives rise to the formation of new bonds proportional to $\Delta N\left(t_{1}\right)$, which in turn, leads to the enhancement of stiffness by $\Delta \hat{C}$. The quantity $\Delta \hat{C}$ can be represented as a sum

$$
\Delta \hat{C}\left(N\left(t_{1}\right), T\left(t_{1}\right)\right)=\Delta \hat{C}_{T}\left(N\left(t_{1}\right), T\left(t_{1}\right)\right)+\Delta \hat{C}_{N}\left(N\left(t_{1}\right), T\left(t_{1}\right)\right),
$$

where $\Delta C_{N}=\partial \hat{C} / \partial N \Delta N ; \Delta C_{T}=d \hat{C} / d t \Delta t-\Delta C_{N}$. The value of the second term is specified by an increase in the stiffness of vitrifying composite due to the formation of new intermolecular bonds in the binding agent. Their unstrained (natural) state coincides with the current (actual) state of the material at the time of their formation (stiffness enhancement without tension (Wang et al.
2001)). Therefore, the increment of the stress tensor can be expressed as

$$
\Delta \sigma\left(t_{1}\right)=\Delta \hat{C}_{T}\left(N\left(t_{1}\right), T\left(t_{1}\right)\right): \overline{\hat{\varepsilon}}\left(t_{1}\right)+\hat{C}\left(N\left(t_{1}\right), T\left(t_{1}\right)\right): \Delta \overline{\hat{\varepsilon}}\left(t_{1}\right),
$$

where $\overline{\hat{\varepsilon}}=\hat{\varepsilon}-\int_{T_{H}}^{T} \hat{\alpha}(N(\tau), T(\tau)) d N(\tau), \hat{\varepsilon}$ is the strain tensor, $\hat{\sigma}$ is the stress tensor, $\Delta \hat{\varepsilon}$ is the increment of the strain tensor until the time of subsequent variation in the rate of conversion of the composite. For arbitrary time $t_{i}$ associated with temperature $T_{g 2}<T\left(t_{i}\right)<T_{g 1}$ and the rate of conversion $0<N\left(t_{i}\right)<1$ the relation $\Delta \sigma=f(\Delta \varepsilon)$ takes the following form

$$
\Delta \hat{\sigma}\left(t_{i}\right)=\hat{C}\left(N\left(t_{i}\right), T\left(t_{i}\right)\right): \overline{\hat{\varepsilon}}\left(t_{i}\right)+\Delta \hat{C}_{T}\left(N\left(t_{i}\right), T\left(t_{i}\right)\right): \Delta \overline{\hat{\varepsilon}}\left(t_{i}\right)
$$

The values of stresses at the arbitrary moment of time $t_{k}$ are determined by summing the prehistory of the process

$$
\hat{\sigma}\left(t_{k}\right)=\sum_{i=0}^{k} \Delta \hat{\sigma}\left(t_{i}\right)
$$

Upon substituting (19) into (20) we obtain using (18) the following equation:

$$
\hat{\sigma}\left(t_{k}\right)=\sum_{i=0}^{k}\left[\begin{array}{c}
\hat{C}\left(N\left(t_{i}\right), T\left(t_{i}\right)\right): \Delta \overline{\hat{\varepsilon}}\left(t_{i}\right) \\
+\Delta \hat{C}\left(N\left(t_{i}\right), T\left(t_{i}\right)\right): \overline{\hat{\varepsilon}}\left(t_{i}\right) \\
-\Delta \hat{C}_{N}\left(N\left(t_{i}\right), T\left(t_{i}\right)\right): \overline{\hat{\varepsilon}}\left(t_{i}\right)
\end{array}\right] .
$$

The implementation of transition to the limit allows us to write the constitutive Eq. (21) in the integral form

$$
\hat{\sigma}(t)=\hat{C}(N(t), T(t)): \overline{\hat{\varepsilon}}(t)-\int_{N(0)}^{N(t)} C_{N}^{\prime}(N(\tau), T(\tau)): \overline{\hat{\varepsilon}}(\tau) d N,
$$

where $\hat{C}_{N}=\partial \hat{C} / \partial N$. Using expansion (18) the expression for the current stiffness of the material is written as

$$
\hat{C}(N(t), T(t))=\hat{C}(0, T(t))+\int_{N(0)}^{N(t)} C_{N}^{\prime}(N(\tau), T(\tau)) d N .
$$

The substitution of this expression into (22) followed by combining like terms allows us to write the constitutive relations in the following form:

$$
\sigma^{c}(t)=\hat{C}(0, T(t)): \overline{\hat{\varepsilon}}^{g}(t)+\int_{0}^{N(t)} \hat{C}_{N}^{\prime}(N(\tau), T(\tau)):\left[\overline{\hat{\varepsilon}}^{g}(t)-\overline{\hat{\varepsilon}}^{g}(\tau)\right] d N .
$$

Since Eq. (23) are valid for the material subject to cooling from $T_{H}>T_{g 1}$, at the initial time the rate of conversion 
is $N(0)=0$ and in (23) the integration limit is entitled to a change. Index "g" denotes glass transition. We will further suppose that after being cooled to temperature $T_{H}<T_{g 2}$, the composite is subject to heating according to deformation scheme (23). The bonds with frozen strains formed in the process of cooling are destroyed. Let at time $t_{i}$ corresponding to the rate of conversion and temperature $T_{i}$ some fraction of bonds $\Delta N_{i}$ be lost due to a rise of temperature. By analogy with (19) the expression for the stress increment up to the moment of subsequent temperature variation is written as

$$
\begin{aligned}
\Delta \hat{\sigma}^{H}=\hat{C} & \left(N\left(t_{i}\right), T\left(t_{i}\right)\right): \Delta \overline{\hat{\varepsilon}}^{h}\left(t_{i}\right) \\
& +\Delta \hat{C}_{T}\left(N_{i}, T\left(t_{i}\right)\right): \overline{\hat{\varepsilon}}^{h}\left(t_{i}\right)+ \\
& +\Delta \hat{C}_{N}\left(N\left(t_{i}\right), T\left(t_{i}\right)\right):\left[\overline{\hat{\varepsilon}}^{h}\left(t_{i}\right)-\hat{\varepsilon}^{g}\left(t_{i}\right)\right] .
\end{aligned}
$$

The strains and strain increments indexed " $h$ "correspond to the stage of heating. The difference comprising the last term in the right-hand side of expression (24) means that disappearance of $\Delta N_{i}$ bonds leads to a release of strains "frozen" during vitrification, which results in a stress jump proportional to the difference of current strains corresponding to $N\left(t_{i}\right)$ in the soften and cured polymers. This can be explained by the fact that the unstrained state of a given number of bonds is identical to the current state of the medium at the time of their formation. Representing $\Delta \hat{C}_{T}$ in terms of $\Delta \hat{C}_{N}, \Delta \hat{C}$ as in (18), expression (24) can be transformed into

$$
\begin{aligned}
\Delta \hat{\sigma}^{H}\left(t_{i}\right)=\hat{C} & \left(N\left(t_{i}\right), T\left(t_{i}\right)\right): \Delta \overline{\hat{\varepsilon}}^{h}\left(t_{i}\right)+ \\
& +\left[\Delta \hat{C}\left(N\left(t_{i}\right), T\left(t_{i}\right)\right)-\Delta \hat{C}_{N}\left(N\left(t_{i}\right), T\left(t_{i}\right)\right)\right]: \Delta \hat{\bar{\varepsilon}}^{h}\left(t_{i}\right)+ \\
& +\Delta \hat{C}_{N}\left(N\left(t_{i}\right), T\left(t_{i}\right)\right):\left[\overline{\hat{\varepsilon}}^{h}\left(t_{i}\right)-\overline{\hat{\varepsilon}}^{g}\left(t_{i}\right)\right]
\end{aligned}
$$

Upon combining like terms and passing first to the integral sum and then to the integral we arrive at the expression for the stress-strain state in a softened composite

$$
\begin{aligned}
\Delta \hat{\sigma}^{H}(t)=\hat{C}(N(t), T(t)): \Delta \widehat{\bar{\varepsilon}}^{h}\left(t_{i}\right)-\int_{1}^{N(t)} \hat{C}_{N}^{\prime}(N(\tau), T(\tau)): \overline{\widehat{\varepsilon}}^{g}(\tau) d N(\tau)- \\
\quad-\int_{0}^{1} \hat{C}_{N}^{\prime}(N(\tau), T(\tau)): \overline{\hat{\varepsilon}}^{g}(\tau) d N(\tau)
\end{aligned}
$$

Combining the two integrals in the right-hand-side of the expression we obtain the final expression for physical relations:
$\Delta \hat{\sigma}^{H}(t)=\hat{C}(N(t), T(t)): \Delta \overline{\hat{\varepsilon}}^{h}\left(t_{i}\right)-\int_{0}^{N(t)} \hat{C}_{N}^{\prime}(N(\tau), T(\tau)): \overline{\hat{\varepsilon}}^{g}(\tau) d N(\tau)$

The stress-strain relations for polymer composites undergoing glass transition, which are derived based on the stiffness-growth method (23), (25), are more general compared to the "energy" relations (12) and therefore more accurate in describing the glass transition processes. However, they are awkward to handle in practice because of the necessity for accurate evaluation of the stiffness tensor derivative with respect to the conversion degree and calculation of tensor relations $\hat{C}(N), \hat{\varepsilon}^{o}(N)$. In numerical computation of thermomechanical characteristics of composite materials this leads to unreasonably high time costs and memory consumption and what is more undesirable to a loss of accuracy in the case of performing calculations for boundary value technological problems due to inevitably rough estimation of the derivative while doing time discretization. Therefore in practice it is more appropriate to use simpler expression such as (12).

Let us define the class of composite materials, for which the "energy" relations (12) give an accurate description, i.e., are coincide with $(23,25)$. To this end, we represent both types of equations in the component-wise form:

The energy-type relations

$$
\begin{aligned}
\sigma_{i j}^{e}(t)= & C_{i j k l}^{(1)}(t)\left[\varepsilon_{k l}(t)-\varepsilon_{k l}^{T 1}(t)\right]+ \\
& +C_{i j k l}^{(2)}(t) \int_{0}^{N(t)}\left[\varepsilon_{k l}(t)-\varepsilon_{k l}^{o}(\tau)-\varepsilon_{k l}^{T 2}(t)+\varepsilon_{k l}^{T 2}(\tau)\right] d N(\tau) .
\end{aligned}
$$

The stiffness-type relations:

$$
\begin{aligned}
\sigma_{i j}^{s}(t)= & C_{i j k l}(N(t), T(t))\left[\varepsilon_{k l}(t)-\varepsilon_{k l}^{T}(N(t), T(t))\right]- \\
& -\int_{0}^{N(t)}\left[\varepsilon_{k l}(\tau)-\varepsilon_{k l}^{T}(N(\tau), T(\tau))\right] C_{N_{i j k l}^{\prime}}^{\prime}(N(\tau), T(\tau)) d N(\tau) .
\end{aligned}
$$

We need to determine the type of the function $C_{i j k l}, \varepsilon_{k l}$, at which $\sigma_{i j}^{e}=\sigma_{i j}^{s}$. To simplify the transformations we assume that in the glass transition range the dependence of the components of elasticity tensors on temperature is negligible compared to a change of stiffness caused by transition of the matrix into a glassy state. To specify the type of function $C_{i j k l}$ providing a change from (27) to (26), it is necessary to equate the right-hand sides of these expressions without taking into account the thermal deformation (according to the problem formulation the contributions of thermal deformation are similar in both expressions and are deleted on their equating). 


$$
\begin{aligned}
& C_{i j k l}^{(1)} \varepsilon_{k l}(t)+C_{i j k l}^{(2)} \varepsilon_{k l}(t) N(t)-C_{i j k l}^{(2)} \int_{0}^{N(t)} \varepsilon_{k l}(\tau) d N(\tau)= \\
& =C_{i j k l}(N(t), T(t)) \varepsilon_{k l}(t)-\int_{0}^{N(t)} \varepsilon_{k l}(\tau) C_{N_{i j k l}^{\prime}}^{\prime}(N(\tau), T(\tau)) d N(\tau)
\end{aligned}
$$

Upon differentiating with respect to time we obtain

$$
\begin{aligned}
& C_{i j k l}^{(1)} \dot{\varepsilon}_{k l}+C_{i j k l}^{(2)}\left(\dot{\varepsilon}_{k l} N+\varepsilon_{k l} \dot{N}\right)-C_{i j k l}^{(2)} \varepsilon_{k l} \dot{N}= \\
& \quad=\dot{C}_{i j k l} \varepsilon_{k l}+C_{i j k l} \dot{\varepsilon}_{k l}-\varepsilon_{k l} \dot{C}_{i j k l}
\end{aligned}
$$

The arguments are dropped out to shorten the expression. Equation (28) is derived bearing in mind that in the glass transition range $\hat{C}_{N}^{\prime}>>\hat{C}_{T}^{\prime}$ and therefore $\hat{C}_{N}^{\prime} \cong$ $d \hat{C} / d t$. After combing like terms expression (28) reduces to

$$
C_{i j k l}=C_{i j k l}^{(1)}+C_{i j k l}^{(2)} N
$$

Thus, to ensure that in the description of behavior of composite material undergoing glass transition the energy-type relations coincide with the stiffness-type relations, the dependence of the components of the elastic tensor on the degree of material conversion should obey the law (29)

In order to obtain the functional dependence of the components of free deformation tensor $\varepsilon_{k l}^{T}(N, T)$, which will provide identity of (26) with (27), it is necessary to equate the right-hand sides of these expressions taking into account the fact that for the specified type of elastic tensor (29) the sum of terms, containing effective strains on each side of the equality sign, are identical.

$$
\begin{aligned}
& -C_{i j k l}^{(1)} \varepsilon_{k l}^{T 1}(t)=C_{i j k l}^{(2)} \int_{0}^{N(t)}\left[\varepsilon_{k l}^{T 2}(t)-\varepsilon_{k l}^{T 2}(\tau)\right] d N(\tau)= \\
& =-\left[C_{i j k l}^{(1)}+C_{i j k l}^{(2)} N(t)\right] \varepsilon_{k l}^{T}(t)+C_{i j k l}^{(2)} \int_{0}^{N(t)} \varepsilon_{k l}^{T}(\tau) d N(\tau)
\end{aligned}
$$

In view of the accepted assumptions, differentiating the above expression with respect to time and collecting like terms leads to a system of linear first-order differential equations with variables separated with respect to $\varepsilon_{k e}^{T}$ :

$$
\dot{\varepsilon}_{k l}^{T}\left[C_{i j k l}^{(1)}+C_{i j k l}^{(2)} N\right]=C_{i j k l}^{(1)} \dot{\varepsilon}_{k l}^{T 1}+C_{i j k l}^{(2)} \dot{\varepsilon}_{k l}^{T 2} N
$$

For transversally isotropic and orthotropic composites the system (30) can be explicitly solved for $\dot{\varepsilon}_{k l}^{T} \equiv d \varepsilon_{k l}^{T} / d t$. In this case, $\{\dot{\varepsilon}\}=[C]^{-1}\left\{\dot{\varepsilon}^{T}\right\}$, where $[C]$ is a $3 \times 3$ square matrix, $C_{i j}=C_{i j k l}^{(1)}+C_{i j k l}^{(2)} N ; \quad\left\{\dot{\varepsilon}^{T}\right\}$ is a column vector, such that $\dot{\varepsilon}_{i}^{T}=C_{i i j j}^{(1)} \dot{\varepsilon}_{j i}^{T 1}+C_{i i j j}^{(2)} \dot{\varepsilon}_{j j}^{T 2} ; \dot{\varepsilon}_{i}^{0}=\dot{\varepsilon}_{i i}^{0}$. Denoting the inverse of the matrix $[C]$ by $[\bar{C}]$ we obtain an explicit expression for components of the simple deformation tensor

$$
\varepsilon_{i i}^{T}=\int_{0}^{t} \bar{C}_{i j} \dot{\varepsilon}_{j}^{T} d t
$$

where

$$
\begin{aligned}
\bar{C}_{11} & =\left[\left(C_{22}\right)^{2}-\left(C_{23}\right)^{2}\right] / \Delta ; \quad \bar{C}_{12}=\left[C_{12}\left(C_{23}-C_{22}\right)\right] / \Delta ; \\
\bar{C}_{23} & =\left[\left(C_{12}\right)^{2}-C_{11} C_{33}\right] / \Delta ; \quad \bar{C}_{22}=\left[C_{11} C_{22}-\left(C_{12}\right)^{2}\right] / \Delta ; \\
\Delta & =2\left(C_{23}\right)^{2}\left(C_{12}-C_{22}\right)+C_{33}\left(\left(C_{22}\right)^{2}-\left(C_{12}\right)^{2}\right) .
\end{aligned}
$$

Expression (31) describes the dependence of temperature deformation on time providing going from the "stiffness growth" relations to "the energy" relations and back.

Expressions (29) (31) allow us to single out the class of composites, for which both approaches give the same results. The most demonstrative example is the composite containing no filling material. In other materials such similarities might be the result of the random combination of matrix and fiber properties. Therefore it is essential to estimate the errors that occur in the calculations due to the application of simplified relations (12). To this end, we carry out a comparative analysis of the "energy" and "stiffness" models with the aim of averaging the thermomchanical properties of the composite material based on the Voigt and Reuss methods.

We assume that the matrix and fiber moduli, as well as their thermal expansion coefficients $\alpha_{M}, \alpha_{a}$ are not temperature dependent. The loading sequence includes the following stages: cooling of the clamped specimen from $T>T_{g 1}$ to $T_{k}>T_{g 2}$; unloading at $T=T_{k}$; re-clamping and heating up to $T_{H}$. According to (26), (27), the stress in the cooled specimen will change by the law (index "g" denotes glass transition, index "e" refers to stresses calculated by the "energy" model (26), :" s" - to stresses calculated by the "stiffness" model (27)):

$$
\begin{aligned}
& \sigma^{e g}(T)=-E^{(1)} \varepsilon^{T 2}-E^{(2)}\left[\varepsilon^{T 2}(T) N(T)-\int_{0}^{N(t)} \varepsilon^{T 2}(T(\tau)) d N(\tau)\right] ; \\
& \sigma^{s g}(T)=-E(N(T)) \varepsilon^{T}(T)+\int_{0}^{N(t)} \varepsilon^{T}(T(\tau)) E_{N}^{\prime}(N(\tau)) d N(\tau) .
\end{aligned}
$$

Unloading and subsequent heating of the material to the initial temperature are accompanied by the generation of stresses (index " $\mathrm{h}$ " denotes heating): 


$$
\begin{aligned}
\sigma^{e h}(T)= & -E^{(1)} \varepsilon^{T 1}(T)-E^{(2)}\left[\varepsilon^{T 2}(T) N+\int_{0}^{N(t)} \varepsilon^{T 2}(T(\tau)) d N(\tau)\right]+ \\
& +\left[E^{(1)}+E^{(2)} N(T)\right] \varepsilon\left(T_{k}\right) ;
\end{aligned}
$$

$$
\sigma^{s h}(T)=E(T)\left[\varepsilon^{s}\left(T_{k}\right)-\varepsilon^{T}(T)\right]+\int_{0}^{N(t)} \varepsilon^{0}(T(\tau)) E_{N}^{\prime}(T(\tau)) d N(\tau),
$$

where $\varepsilon\left(T_{k}\right)$, is the strain in the cooled specimen after unloading: $\varepsilon\left(T_{k}\right)=-\sigma^{e g}\left(T_{k}\right) /\left(E^{(1)}+E^{(2)}\right) ; \quad \varepsilon^{s}\left(T_{k}\right)=-\sigma^{s g}\left(T_{k}\right) /$ $E\left(T_{k}\right)$. The values of the effective thermomechanical characteristics in (32)-(35) depend on the method of averaging the properties of the composite.

Consider the process of specimen deformation along the fiber length, the computational scheme of which is shown in Fig. 2a. In this case $\sigma=\sigma_{a} \psi+\sigma_{M} \bar{\psi} ; \quad \varepsilon_{a}=\varepsilon_{M}=\varepsilon$. Index " $M$ " refers to the matrix and "a" - to the reinforcement. The effective parameters are determined from the following formulas

$$
\begin{aligned}
\varepsilon(N) & =E_{M} \bar{\psi}+E_{a} \psi ; \\
\varepsilon^{T} & =\int_{T_{H}}^{T}\left(\bar{\psi} \alpha_{M} E_{M}+\psi E_{a} \alpha_{a}\right)\left(E_{M} \bar{\psi}+E_{a} \psi\right)^{-1} d t ; \\
E^{(1)} & =E_{M}^{(1)} \bar{\psi}+E_{a} \psi ; \quad E^{(2)}=E_{M}^{(2)} \psi ; \\
\varepsilon^{T 1} & =\int_{T_{H}}^{T}\left[E_{M}^{(1)} \alpha_{M} \bar{\psi}+E_{a} \alpha_{a} \psi\right]\left[E_{M}^{(1)} \bar{\psi}+E_{a} \psi\right]^{-1} d T ; \\
\varepsilon^{T 2} & =\int_{T_{H}}^{T} \alpha_{M} d T,
\end{aligned}
$$

Here $E_{M}^{(1)}$ is the equilibrium modulus of the binding agent; $E_{M}^{(2)}=E_{M}^{g}-E_{M}^{(1)} ; E_{a}, \alpha_{a}, E_{M}, \alpha_{M}$ are the moduli and liner coefficients of thermal expansion of reinforcing and binding materials, respectively; $E_{M}=E_{M}^{(1)}+E_{M}^{(2)} N$; $\bar{\psi}=1-\psi$.

It turns out that upon substituting the "stiffness" and "energy" relations into (32)-(35) we obtain the same values of stresses:

$$
\begin{aligned}
& \sigma_{/ /}^{e g}(T)=\sigma_{/ /}^{s g}(T)=-\int_{T_{H}}^{T(t)}\left[E_{M}^{(1)}+E_{M}^{(2)} N(\tau) \bar{\psi} \alpha_{M}+E_{a} \psi \alpha_{a}\right] d T(\tau) ; \\
& \sigma_{/ /}^{e h}(T)=\sigma_{/ /}^{s h}(T)=\varepsilon\left(T_{k}\right)\left[\bar{\psi} E_{M}+\psi E_{a}\right]+\sigma_{/ /}^{e g}(T),
\end{aligned}
$$

where $\varepsilon\left(T_{k}\right)=-\sigma^{e g}\left(T_{k}\right)\left(\bar{\psi}\left(E_{M}^{(1)}+E_{M}^{(2)}\right)+\psi E_{a}\right)^{-1}$.

Exact effective stresses are calculated by solving the problem of deformation behavior of a composite, in which the matrix and fiber deform simultaneously. The system of equations for this case includes the constitutive equations, equilibrium equations and equations of deformation compatibility:

$$
\begin{aligned}
& \left.\sigma_{M}(T)=E_{M}(T) \varepsilon_{M}^{T}(T)-E_{M}^{(2)} \int_{0}^{N(t)} \varepsilon_{M}^{T}(\tau) d N(\tau) ; \sigma_{a}(T)=E_{a} \varepsilon_{a}^{T}(T) ;\right\} \\
& \sigma(37) \\
& =\sigma_{a}(T) \psi+\sigma_{M}(T) \bar{\psi} ; \quad \varepsilon(T)=\varepsilon_{a}(T)=\varepsilon_{M}(T) .
\end{aligned}
$$

Solving Eqs. (37) and (38) taking into account the deformation conditions and temperature variation, we obtain the effective stresses that agree with (36). Thus, for the specified computational scheme both the "energy" and "stiffness" relations yield an exact solution.

No such agreement is observed in the case of deformation across the fiber (Reuss's average, Fig. 2b). In this case, the effective characteristics of the material are found by the following formulas:

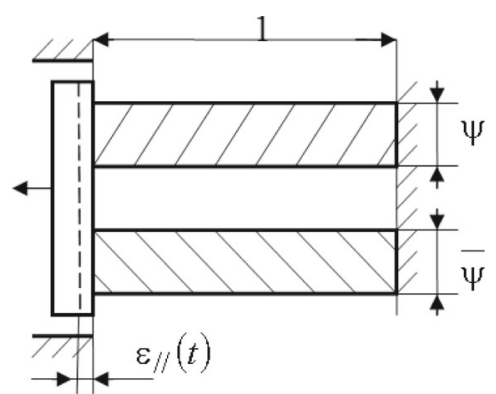

b

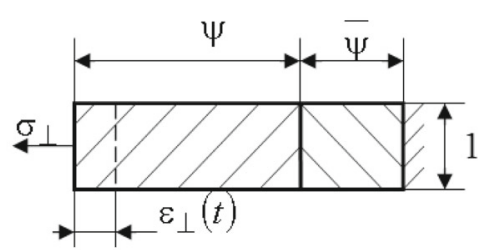

Fig. 2 Schematic representation of composite deformation: a- along the fiber, $\mathbf{b}$ - across the fiber 


$$
\begin{aligned}
E(N) & =E_{M} E_{a}\left[E_{a} \bar{\psi}+E_{M} \psi\right]^{-1} ; \\
\varepsilon^{T} & =\int_{T_{H}}^{T}\left(\alpha_{M} \bar{\psi}+\alpha_{a} \psi\right) d T ; \\
E^{(1)} & =E_{M}^{(1)} E_{a}\left[E_{a} \bar{\psi}+E_{M}^{(1)} \psi\right]^{-1} ; \\
E^{(2)} & =\left[E_{M}^{(1)}+E_{M}^{(2)}\right] E_{a}\left[E_{a} \bar{\psi}+\left(E_{M}^{(1)}+E_{M}^{(2)}\right)\right]^{-1}-E^{(1)} ; \\
\varepsilon^{T 1} & =\varepsilon^{T 2}=\int_{T_{H}}^{T}\left(\alpha_{M} \bar{\psi}+\alpha_{a} \psi\right) d T ; \quad E_{M}=E_{M}^{(1)}+E_{M}^{(2)} N .
\end{aligned}
$$

Substituting these expressions into (32)-(35), we get

$$
\left.\begin{array}{rl}
\sigma_{\perp}^{e g}(T) & =-\int_{T_{H}}^{T}\left[E^{(1)}+E^{(2)} N(T)\right]\left[\alpha_{M} \bar{\psi}+\alpha_{a} \psi\right] d T ; \\
\sigma_{\perp}^{e h}(T) & =\left(E^{(1)}+E^{(2)} N\right) \varepsilon^{e}\left(T_{k}\right)+\sigma^{e g}(T) ;
\end{array}\right\}
$$

where $\varepsilon^{e}\left(T_{k}\right)=-\sigma^{e g}\left(T_{k}\right)\left[E^{(1)}+E^{(2)}\right]^{-1} ; \quad \varepsilon^{s}\left(T_{k}\right)=-\sigma^{s g}\left(T_{k}\right)$ $\left[\bar{\psi}\left(E_{M}^{(1)}+E_{M}^{(2)}\right)+\psi E_{a}\right]^{-1}$.

An exact solution is found by solving the system (36) bearing in mind that

$$
\begin{aligned}
\sigma(T) & =\sigma_{a}(T)=\sigma_{M}(T) ; \quad \varepsilon(T) \\
& =\varepsilon_{a}(T) \psi+\varepsilon_{M}(T) \bar{\psi}
\end{aligned}
$$

Upon substituting (41) into (36) and making some transformations we obtain the expressions for stresses, which agree with the "stiffness" expressions $(40)$. Hence, the "stiffness" relations describe accurately the macrobehavior of the composite material for simple variants of averaging the composite properties. The "energy" equations lead to an error in the stress estimates in the case of transverse deformation. Figure 3 presents the reduced stress curves obtained from numerical calculations using (36), (39), (40).

Curves 1,2 describe variation of stresses in the specimen stretched under tensile loads along the fiber length. The characteristics of tested composite material roughly agree with the characteristics of anisotropic fiber - reinforced organoplastic material reinforced with anisotropic fibers the transverse modulus of which is comparable with the matrix modulus. Curves 3-5 for stresses in transversely loaded material at different values of $\psi$ are shown in Fig. 3. The dynamics of the relative error $\Delta \bar{\sigma}$ is presented in Fig. 4.

It is seen that the maximum error is observed in the glass transition interval. Its value increases with the

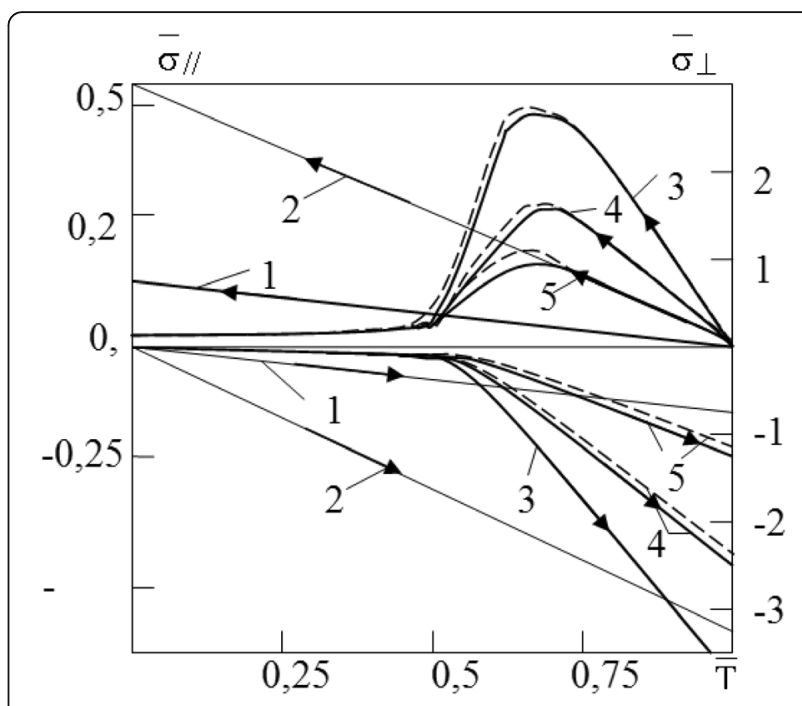

Fig. 3 Reduced stresses in composites: 1,2 - longitudinal stresses: $E_{m}^{(1)}$ $=10^{-4}, E_{m}^{(2)}=10^{-2} ; \psi=0,1(1), 0,5(2) ; 3-5$-transverse stresses: $E_{m}^{(1)}=$ $0,01, E_{m}^{(2)}=1 ; \psi=0,1$ (3), 0,5 (4), 0,7 (5). Solid lines - calculations using (39); dash lines - calculations using (40). The notation used in Fig. 3

and 4: $\bar{\sigma}=\sigma /\left(a_{a} E_{a} \theta_{k}\right) ; \quad \bar{E}_{M}^{(i)}=E_{M}^{(i)} / E_{a} ; \quad \bar{a}_{M}=a_{M} / a_{a} ;$

$\theta k=T_{H}-T_{k} ; \quad \theta_{g}=T_{H}-T_{g} ; \Delta \bar{\sigma}=2\left|\bar{\sigma}^{e}(T)-\bar{\sigma}^{s}(T) \| \sigma^{e}\left(T_{k}\right)-\sigma^{s}\left(T_{k}\right)\right|^{-1} ;$ $\bar{T}=\left(T_{H}-T\right) / \theta_{k}$

growth of $\psi$ and reaches its maximum value at $\psi=0,96$. In the temperature range corresponding to the operating conditions of the end product (lower than $T_{g 2}$ ), the calculation error does not exceed $10 \%$. For polymer materials reinforced with isotropic fibers (glass-, boronplastics, etc.) with $E_{M^{(2)}}<<1$ the maximum error in the estimates of transverse stresses is as high as $3 \%$ (Fig. $4 \mathrm{~b}$, curve 1). This suggests that the "energy" relations can be readily used for description of the behavior of composite materials in the examined range of material properties

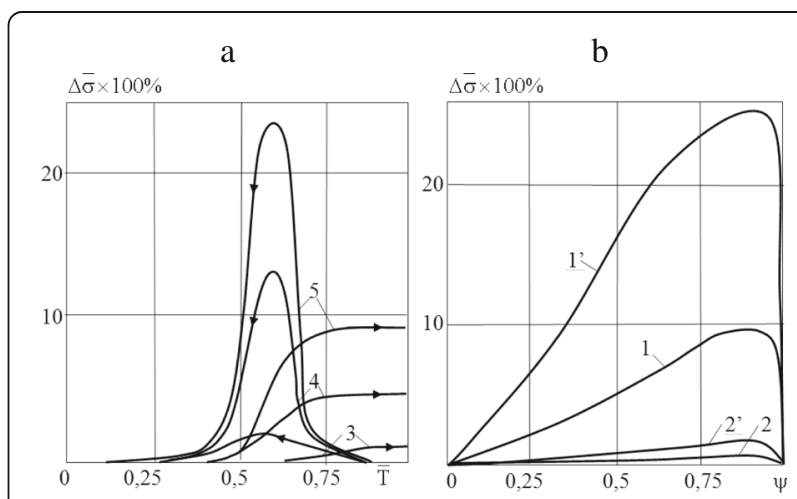

Fig. 4 Errors of stress calculation by (39) as a function of temperature (a) (notation is similar to that used in Fig. 3) and (b) of volume fraction of fibers. $1-E_{M}^{(1)}=0,001, E_{M}^{(2)}=1 ; 2-E_{M}^{(1)}=10^{-4}, E_{M}^{(2)}=10^{-2} ; 1^{\prime}, 2^{\prime}-$ the same relations under heating conditions 
reducing thereby the computational costs related with the evaluation of unknown thermomechanical constants.

\section{Results and discussion}

Predicting of the effective material functions and constants for fiber-reinforced composite based on the results of numerical experiments

One of the challenging problems in the mechanics of composite materials is the estimation of their mechanical properties based on the properties of their components. In our case it is necessary to determine two sets of parameters, i.e., the parameters corresponding to the high-elastic state of the binder $-C_{i j k l}^{(1)}, \alpha_{i i}^{(1)}$, and the parameters corresponding to the glassy state $-C_{i j k l}^{g}, \alpha_{i i}^{g}$.

Consider the unlimited, continuously reinforce medium whose structure is formed by straightened and equally oriented cylindrical fibers having equal circular cross sections. The space between the fibers is filled with the binding medium - the matrix whose characteristics differ from the characteristics of the fiber.

The emphasis is on the prediction of the effective properties of the idealized homogeneous medium based on the properties of the phases and their geometrical characteristics, using for this purpose the averaging procedure.

To this end, we pick out from the medium a unit symmetry cell (we will consider a hexagonal ordering of fibers) - an elementary unit (Fig. 5). We assume that within the boundaries of this unit cell the gradient of the external force field changes insignificantly.

The volume fraction of fibers in the composite is defined by the radius of inclusion: $\psi=\frac{2 V_{s}}{V_{c}}=\frac{2 c \pi R^{2}}{4 a b c}=\frac{\pi R^{2}}{2 a b}$. Hence $R$ $=\sqrt{\psi \frac{2 a b}{\pi}}$. Since we consider a hexagonal cell, we have $a=\frac{b}{\sqrt{3}}$

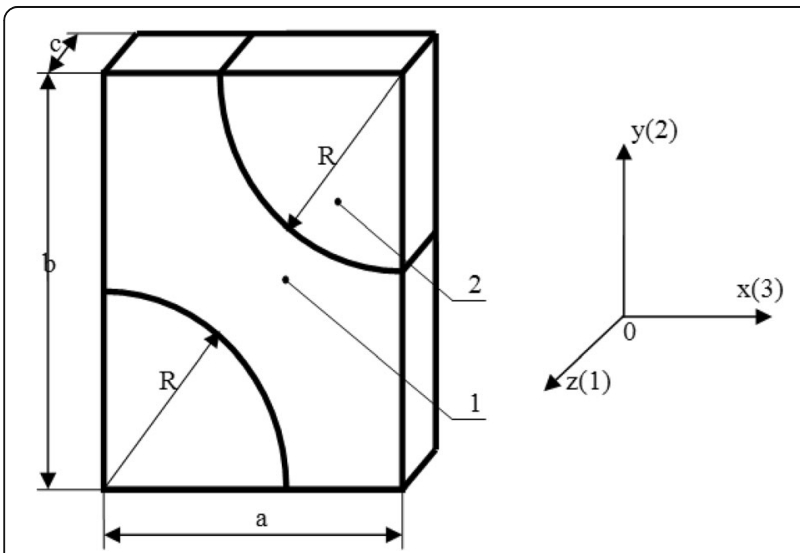

Fig. 5 Periodicity cell and accepted coordinate system:1- matrix of the composite, 2- filler (fiber)
The general formulation of the problem of the linear elasticity theory is reduced to the following system of equations:

equilibrium equation

$$
\sigma_{i j, j}^{*}(\mathbf{x})=0
$$

geometrical relations

$$
\varepsilon_{i j}^{*}(\mathbf{x})=\frac{1}{2}\left(u_{i, j}^{*}(\mathbf{x})+u_{j, i}^{*}(\mathbf{x})\right)
$$

physical relations for polymer matrix

$$
\sigma_{i j}^{*}(\mathbf{x})=\left[B^{m}-\frac{2}{3} G^{m}\right] \theta^{*} \delta_{i j}-2 G^{m} \varepsilon^{*}{ }_{k l}-3 B^{m} \varepsilon_{T}^{m} \delta_{i j},
$$

- physical relations for fiber

$$
\sigma_{i j}^{*}(\mathbf{x})=C_{i j k l}^{a}(T)\left[\varepsilon_{k l}-\varepsilon_{T k l}^{a}(T)\right],
$$

Here $\sigma_{i j}^{*}(\mathbf{x}), \varepsilon^{*}{ }_{i j}(\mathbf{x})$, are the components of the strain and stress tensors at the structural level. The elastic moduli of the matrix $G^{m}, B^{m}$ are taken to be $G_{1}, B_{1}$ in the high-elastic state and $G_{g}, B_{g}$ - in the glassy state.

The boundary conditions depend on what components of the elasticity and temperature deformation tensors we need to determine.

The effective properties describe and specify the deformation of the composite material at the macroscopic level when the composite is considered to be a homogeneous material. The stress state of the elementary macro-units is characterized by the averaged stress tensor $\sigma_{i j}$, and the strain state - by the averaged strain tensor $\varepsilon_{i j}$, which in the case of their slow variation from point to point and the presence of rigid bonds between the components of the composite is determined by the stress-strain state in the elements of the composite structure .

$$
\sigma_{i j}=\frac{1}{V} \int_{V} \sigma_{i j}^{*}(\mathbf{x}) d \mathbf{x}, \varepsilon_{i j}=\frac{1}{V} \int_{V} \varepsilon^{*}{ }_{i j}(\mathbf{x}) d \mathbf{x} .
$$

The generalized Hook law for macroscopic quantities can be written as

$$
\sigma_{i j}=C_{i j m n} \varepsilon_{m n}
$$

where $C_{i j m n}$ is the tensor of the effective elasticity moduli.

Consider a composite material consisting of the isotropic matrix and transversely-isotropic fibers. The composite is assumed to be transversely isotropic such that the isotropy axis coincides with the fiber direction ( $\mathrm{z}$ axis (1)) and the $x(3)-y(2)$-plane is referred to as the isotropy plane. 
A transversely isotropic (monotropic) material $C_{i j k l}$ has five independent components

$$
C_{i j k l}=\left[\begin{array}{cccccc}
C_{1111} & C_{1122} & C_{1122} & 0 & 0 & 0 \\
& C_{2222} & C_{2233} & 0 & 0 & 0 \\
& & C_{2222} & 0 & 0 & 0 \\
& & & \frac{1}{2}\left(C_{2222}-C_{2233}\right) & 0 & 0 \\
& & & & C_{6666} & 0 \\
& & & & & C_{6666}
\end{array}\right]
$$

The physical equations of elasticity for transversely isotropic materials are written as

$$
\begin{aligned}
& \varepsilon_{11}=\frac{1}{E_{1}} \sigma_{11}-\frac{\nu_{12}}{E_{1}} \sigma_{22}-\frac{\nu_{12}}{E_{1}} \sigma_{33}, \\
& \varepsilon_{22}=-\frac{v_{12}}{E_{1}} \sigma_{11}+\frac{1}{E_{2}} \sigma_{22}-\frac{\nu_{23}}{E_{2}} \sigma_{33}, \\
& \varepsilon_{33}=-\frac{v_{12}}{E_{1}} \sigma_{11}-\frac{\nu_{23}}{E_{2}} \sigma_{22}+\frac{1}{E_{2}} \sigma_{33}, \\
& \varepsilon_{12}=\frac{1}{2 G_{12}} \sigma_{12}, \\
& \varepsilon_{13}=\frac{1}{2 G_{12}} \sigma_{13}, \\
& \varepsilon_{23}=\frac{1+v_{23}}{E_{2}} \sigma_{23}
\end{aligned}
$$

where $E_{1}, E_{2}, v_{12}, v_{23}, G_{12}$ are five independent effective characteristics of the material.

These characteristic can be found by solving four boundary value problems of the elasticity theory: uniaxial tension along the fiber length, transverse deformation in the plane normal to the fiber direction, shear deformation in the direction of the fiber axis and free temperature deformation.

Under conditions of uniaxial stretching in the fiber axial direction we assume that the periodicity conditions correspond to the following conditions at the cell boundaries:

$$
\begin{aligned}
& \left.u_{1}^{*}\right|_{z=0}=\left.u_{2}^{*}\right|_{y=0}=\left.u_{3}^{*}\right|_{x=0}=0 ;\left.u_{1}^{*}\right|_{z=c}=U_{1}^{0} ; \\
& \left.u_{2}^{*}\right|_{y=b}=\text { const }=U_{2} ;\left.u_{3}^{*}\right|_{x=a}=\text { const }=U_{3} .
\end{aligned}
$$

The value of $U_{1}^{0}$ is specified, and $U_{2}$ and $U_{3}$ are the unknown parameters found by solving the problem. The

Table 1 Elastic characteristics of the composite components

\begin{tabular}{llllll}
\hline & $E_{1}$ & $E_{2}$ & $v_{12}$ & $v_{23}$ & $G_{12}$ \\
\hline Glass fiber & $9,32 E+10$ & - & 0,24 & - & - \\
Boron fiber & $3,70 E+11$ & - & 0,15 & - & - \\
Carbon fiber & $2,26 E+11$ & $1,29 E+10$ & 0,31 & 0,2 & $6,00 E+10$ \\
Organic fiber $\left(T>T_{g}\right)$ & $6,10 E+10$ & $1,60 E+09$ & 0,27 & 0,27 & $1,30 E+09$ \\
Organic fiber $\left(T<T_{g}\right)$ & $1,21 E+11$ & $3,35 E+09$ & 0,27 & 0,27 & $2,60 E+09$ \\
\hline
\end{tabular}

Table 2 Coefficient of linear thermal expansion of fibers

\begin{tabular}{lll}
\hline & $a_{1}, \mathrm{~K}^{-1}$ & $\mathrm{a}_{2}, \mathrm{~K}^{-1}$ \\
\hline Glass fiber & $5 \mathrm{E}-6$ & $5 \mathrm{E}-6$ \\
Carbon fiber & $-5 \mathrm{E}-7$ & $2.7 \mathrm{E}-5$ \\
Organic fiber & $-6,3 \mathrm{E}-6$ & $8,5 \mathrm{E}-5$ \\
\hline
\end{tabular}

composite deformation in the longitudinal direction is constant and equal to $\varepsilon_{11}=\varepsilon=U_{1}^{0} / c$. From the solution of the problem we determine the average longitudinal stresses $\sigma_{11}$ and average transverse strains $\varepsilon_{22}=U_{2} / b$ and $\varepsilon_{33}=U_{3} / a$.

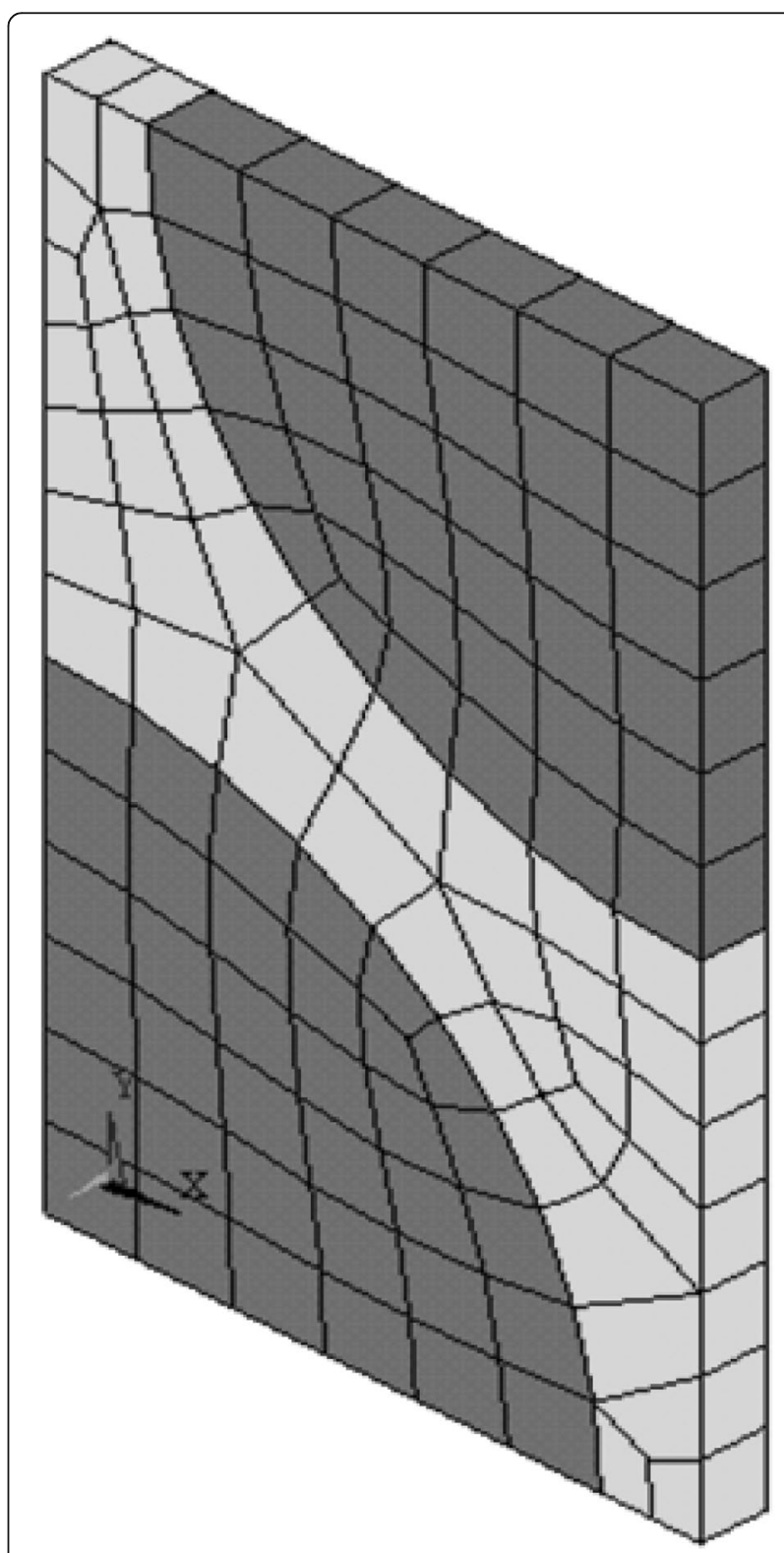

Fig. 6 Finite element discretization of the symmetry cell 

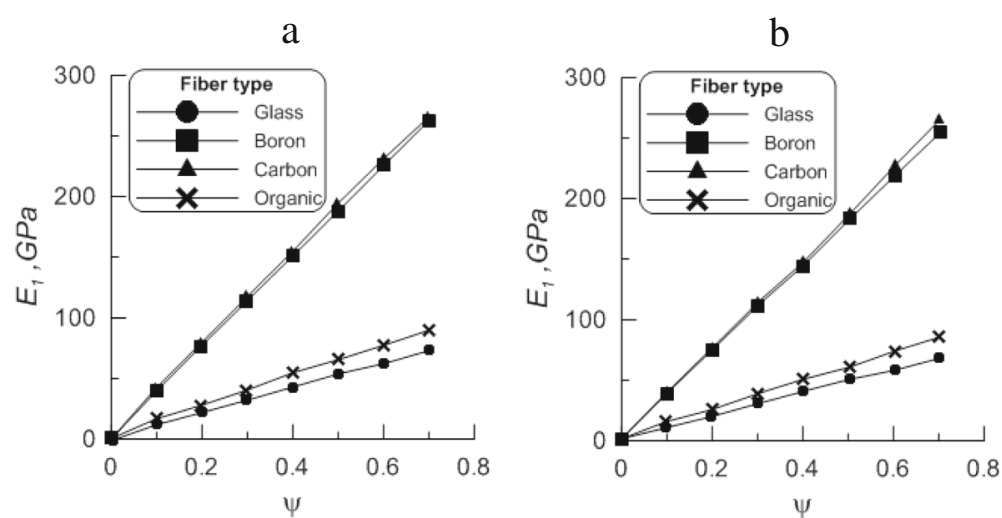

Fig. 7 Plots of longitudinal modulus of the composite against volume fraction of the fiber in (a) the glassy state and (b) in the high elastic state of the matrix

The effective characteristics are defined by the following relations:

$$
E_{1}=\frac{\sigma_{11}}{\varepsilon} ; v_{12}=-\frac{\varepsilon_{22}}{\varepsilon} ; v_{13}=-\frac{\varepsilon_{33}}{\varepsilon} .
$$

For transverse deformations the boundary conditions are written as

$$
\begin{aligned}
& \left.u_{1}^{*}\right|_{z=0}=\left.u_{2}^{*}\right|_{y=0}=\left.u_{3}^{*}\right|_{x=0}=0 ;\left.u_{1}^{*}\right|_{z=c}=U_{1} ; \\
& \left.u_{2}^{*}\right|_{y=b}=\text { const }=U_{2}^{0} ;\left.u_{3}^{*}\right|_{x=a}=\text { const }=U_{3} .
\end{aligned}
$$

The value of $U_{2}^{0}$ is specified, and $U_{1}$ and $U_{3}$ are the unknown parameters found by solving the problem. The composite deformation in the longitudinal direction is constant and equal to $\varepsilon_{22}=U_{2}^{0} / b$. From the solution of the problem we determine the average transverse normal stresses $\sigma_{22}$ and average strains $\varepsilon_{11}=U_{1} / c$ and $\varepsilon_{33}=U_{3} / a$.

The effective characteristics are defined by the following relations:

$$
E_{2}=\frac{\sigma_{22}}{\varepsilon_{22}} ; v_{23}=-\frac{\varepsilon_{33}}{\varepsilon_{22}} ; v_{21}=-\frac{\varepsilon_{11}}{\varepsilon_{22}} .
$$

For simple longitudinal shear we specify the following boundary conditions:

$$
\begin{aligned}
& \left.u_{1}^{*}\right|_{z=0}=\left.u_{2}^{*}\right|_{z=0}=0 ;\left.u_{1}^{*}\right|_{z=c}=U_{1}^{0} ;\left.u_{2}^{*}\right|_{y=b}=\text { const }=U_{2} ; \\
& \left.u_{3}^{*}\right|_{x=0}=0 .\left.u_{3}^{*}\right|_{x=a}=\text { const }=U_{3} .
\end{aligned}
$$

and also the condition of equal displacements of opposite points on the front and rear faces of the cell

$$
u_{i}^{*}(x, y, c)=u_{i}^{*}(x, y, 0) .
$$

The value of $U_{1}^{0}$ is specified, and $U_{2}$ and $U_{3}$ are the unknown parameters found by solving the problem. The shear strain of the composite is equal to $\varepsilon_{12}=U_{1}^{0} / b$. From the solution of the problem we determine the average tangential stresses $\sigma_{11}$ and average transverse

\section{a}

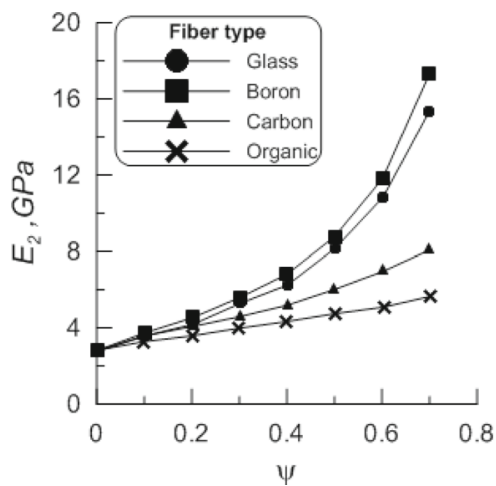

$\mathrm{b}$

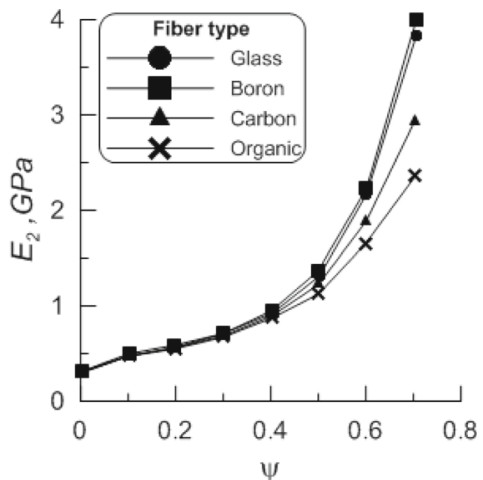

Fig. 8 Plots of transverse modulus of the composite against volume fraction of the fiber in (a) the glassy state and (b) in the high elastic state of the matrix 


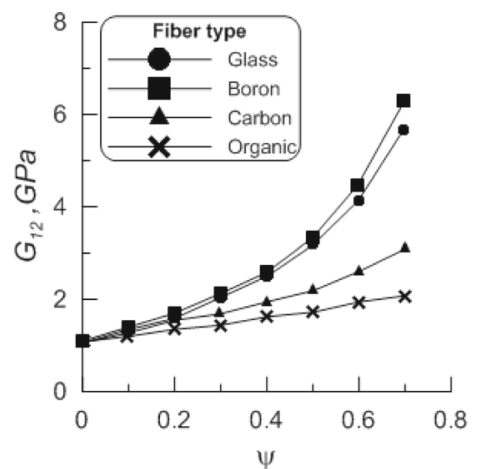

b

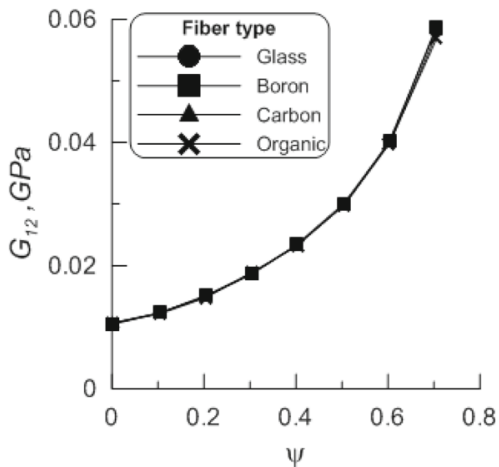

Fig. 9 Plots of shear modulus of the composite against volume fraction of the fiber in (a) the glassy state and (b) in the high elastic state of the matrix

strains $\varepsilon_{22}=U_{2} / b$ and $\varepsilon_{33}=U_{3} / a$. The effective shear modulus is defined as

$$
G_{12}=\frac{\sigma_{12}}{\varepsilon_{12}}
$$

To investigate thermal loading we need to set the temperature deformation $\varepsilon_{T i j}^{m}=\alpha^{m} \Delta T \delta_{i j}$ for the polymer matrix and $\varepsilon_{T i j}^{a}=\alpha_{i j}^{a} \Delta T$ for the reinforcing fiber. Boundary conditions are given as

$$
\begin{aligned}
\left.u_{1}^{*}\right|_{z=0} & =\left.u_{2}^{*}\right|_{y=0}=\left.u_{3}^{*}\right|_{x=0}=0 ;\left.u_{1}^{*}\right|_{z=c}=U_{1} ;\left.u_{2}^{*}\right|_{y=b} \\
& =\text { const }=U_{2} ;\left.u_{3}^{*}\right|_{x=a}=\text { const }=U_{3} .
\end{aligned}
$$

The quantities $U_{1}, U_{2}$ and $U_{3}$ are the unknown parameters found by solving the problem. The average strains $\varepsilon_{11}=U_{1} / a \varepsilon_{22}=U_{2} / b$ and $\varepsilon_{33}=U_{3} / c$ are found by solving the problem The effective coefficients of expansion are derived from

$$
\frac{\alpha_{i i}=\varepsilon_{i i}}{\Delta T}
$$

The stated problems are solved by the finite element method using the ANSYS package. For this purpose the symmetry cell is divided into finite elements It is also necessary to specify the properties of the composite (Tables 1 and 2), boundary conditions and additional conditions of symmetry.

For numerical simulation we used the finite element mesh shown in Fig. 6. We have calculated the thermomechanical characteristics of the composites (glass-, boron-, carbon- and organic plastics) and plotted their properties against the volume fraction of the fiber in the composite (Figs. 7, 8 and 9). The mechanical properties of the binder-containing composite in the glassy state are shown on the left-hand graph and in the high-elastic state - on the right-hand graph.

From the comparison of the figures it is evident that the character of the dependence of all parameters on the a

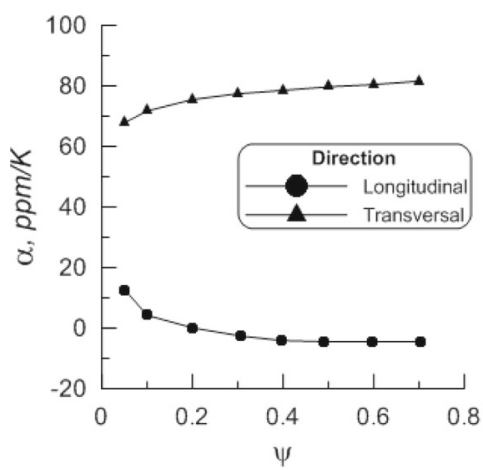

b

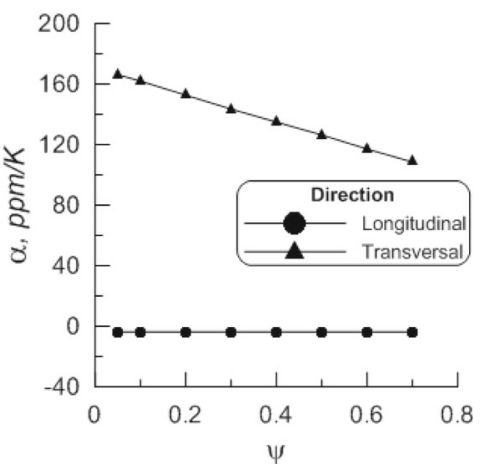

Fig. 10 Plots of thermal expansion coefficients of organoplastic against volume fraction of the fiber in (a) the glassy state and (b) in the high elastic state of the matrix 


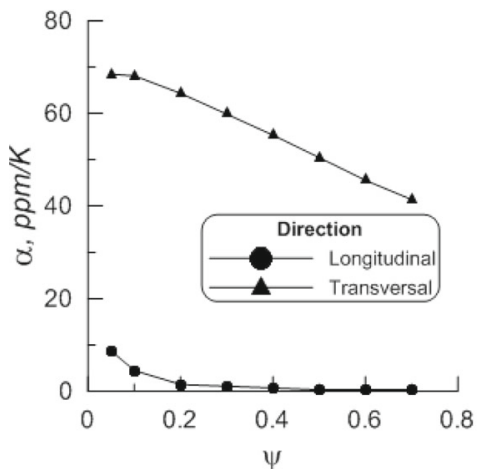

b

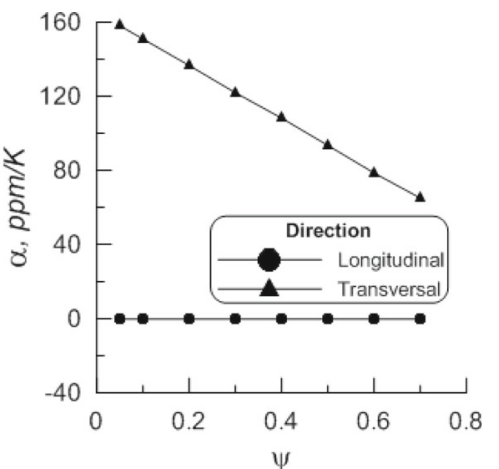

Fig. 11 Plots of thermal expansion coefficients of carbonplastic against volume fraction of the fiber in (a) the glassy state and (b) in the high elastic state of the matrix

coefficient of the volume fraction of fiber remains practically unchanged. Softening of the binding agent in the high-elastic state has negligible effect on the longitudinal modulus of the composite (Fig. 7), but leads to a considerable decrease of the transverse and shear moduli, as well as to weakening of the influence of fiber properties on these quantities (Figs. 8 and 9).

Figures 10, 11 and 12 display the results of calculation of thermal expansion coefficients for organo-, carbon-, and glass-fiber plastics in the glassy and high-elastic state.

To sum up, we can say that the proposed two approaches can be used to construct the constitutive relations in the form of (12) for composites undergoing glass transition. The effective characteristics of the examined composites in the steady states (high-elastic and glassy) can be conveniently predicted based on the known properties of the matrix and fiber.

\section{Conclusion}

- The model of polymer behavior at glass transition recently developed by the authors was generalized to the case of fiber-reinforced polymer matrix composites using two approaches: one is base on the concept of free specific energy, the other on the growth of marix stiffness. The analysis has shown that for homogeneous materials these two approaches are of equal worth, whereas for composite materials they give different results under deformation in the transverse direction.

- The stiffness growth approach is more accurate, because it takes into account the anisotropy of the influence of the degree of conversion on the composite stiffness. However its application involves large computational costs. Moreover, it is highly sensitive to the experimental data errors. a

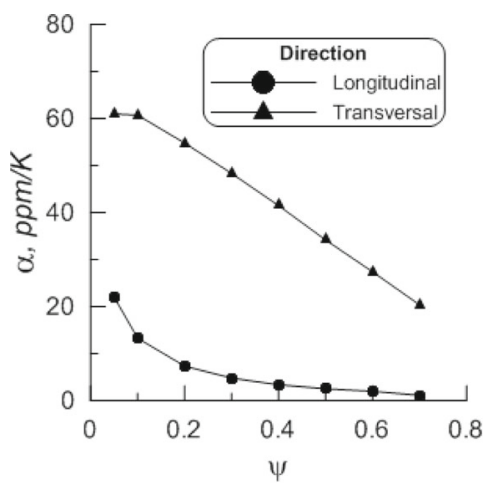

b

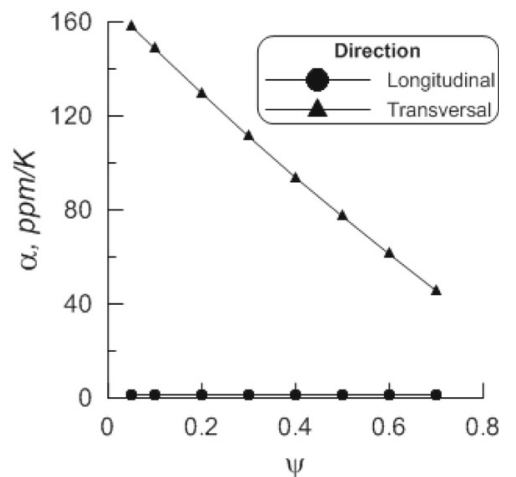

Fig. 12 Plots of thermal expansion coefficients of glass-fiber plastic against volume fraction of the fiber in (a) the glassy state and (b) in the high elastic state of the matrix 
- For composites under longitudinal deformation (the load is applied along the fiber axis) the two approaches agree fairly well. In the case of transverse deformation (the load is applied transverse to the fiber axis) the maximal difference is observed in the glass transition temperature range. In the range of temperatures corresponding to operating conditions of the end product this difference is inessential.

- Using the finite element method and averaging technique a numerical algorithm for calculating the thermoelastic constants of composites in the glassy and high-elastic states has been developed. The thermomechanical properties of the fiber and matrix and volume fraction of fibers are used as the input data.

- The longitudinal, transverse and shear moduli of composites containing different types of fibers have been plotted against the volume fraction of the fiber in the case when the matrix is in the glassy or highelastic state. It has been shown that softening of the matrix has an insignificant effect on the longitudinal modulus of a composite but leads to a considerable decrease of the transverse and shear moduli.

- The coefficients of thermal expansion of composites containing different types of fibers have been plotted against the volume fraction of the fiber in the case when the matrix is in the glassy or high-elastic state. It has been shown that the coefficient of thermal expansion in the transverse direction is much higher than the coefficient of thermal expansion in the longitudinal direction, especially when the composite is in the high-elastic state.

\section{Acknowledgements}

This article was prepared under a grant of Russian Foundation for Basic Research \#16-01-00474.

\section{Authors' contributions}

VPM - Methods, Abstract. NAT - Methods, Conclusion. OYS - Methods, Results, Conclusion. INS - Methods, Results. INV - Translate, Editing. All authors read and approved the final manuscript.

\section{Competing interests}

00000: Materials Science, general

14026: Computational Science and Engineering

18000: Ceramics, Glass, Composites, Natural Materials

\section{Author details}

${ }^{1}$ Institute of Continuum Media Mechanics, Perm, Russian Federation. ${ }^{2}$ Perm National Research Polytechnic University, Perm, Russian Federation.

Received: 7 October 2016 Accepted: 9 November 2016

Published online: 25 November 2016

\section{References}

Askadskiy AA (1973) Deformation of polymers. Chemistry, Moscow Bartenev GM, Zelenev Yu V (1976) Course of lectures in physics of polymers. Chemistry, Moscow
Begishev VP, Matveenko VP, Pistsov NV, Shardakov IN (1997) Modeling of thermomechanical processes in crystallizing polymers. Proceedings of RAS. Solid Mechanics (4):120-132

Brader JM, Voigtmann T, Fuchs M, Larson RG, Cates ME (2009) Glass rheology: From mode-coupling theory to a dynamical yield criterion. Proc Natl Acad Sci U S A 106(36):15186-15191

Buckley CP, Jones DC (1995) Glass-rubber constitutive model for amorphous polymers near the glass transition. Polymer 36:3301-3312

Bugakov II (1989) Constitutive Equations for Materials with Phase Transition. Mech Solids 3:111-117

Christensen RM (1979) Mechanics of composite materials. Wiley-Interscience, New York

Dinzart F, Lipiński P (2010) Self-consistent approach of the constitutive law of a two-phase visco-elastic material described by fractional derivative models. Arch Mech 62(2):135-156

Dupaix RB, Boyce MC (2006) Constitutive modeling of the finite strain behavior of amorphous polymers in and above the glass transition. Mech Mater 39:39-52

Ge Q, Luo X, Rodriguez ED, Zhang X, Mather PT, Dunn ML, Qi HJ (2012) Thermomechanical behavior of shape memory elastomeric composites. J Mech Phys Solids 60:67-83

Ge Q, Luo X, Iversen CB, Nejad HB, Mather PT, Qi HJ (2014) A finite deformation thermomechanical constitutive model for triple shape polymeric composites based on dual thermal transitions. Int J Solids Struct 51:2777-2790

Henann D, Anand L (2008) A constitutive theory for the mechanical response of amorphous metals at high temperatures spanning the glass transition temperature: application to microscale thermoplastic forming. Acta Mater 56:3290-3305

Hill R (1964a) Theory of mechanical properties of fibre-strengthened materials: I. Elastic behaviour. J Mech Phys Solid 12(4):199-212

Hill R (1964b) Theory of mechanical properties of fibre-strengthened materials: Il. Inelastic behaviour. J Mech Phys Solids 12(4):213-218

Hill R (1965a) Theory of mechanical properties of fibre-strengthened materials: III. Self-consistent model. J Mech Phys Solids 13(4):189-198

Hill R (1965b) A self-consistent mechanics of composite materials. J Mech Phys Solids 13(4):212-222

Hill R (1966) Theory of mechanical properties of fiber-reinforced composite materials. Mech Collec Translat Articles 96(2):131-149

Liu YP, Gall K, Dunn ML, Greenberg AR, Diani J (2006) Thermomechanics of shape memory polymers: uniaxial experiments and constitutive modelling. Int J Plast 22(2):279-313

Lustig SR, Shay RM Jr, Caruthers JM (1996) Thermodynamic constitutive equations for materials with memory on a material time scale. J Rheol 40(1):69-106

Malmeister AA, Tamuzh VP, Teters GA (1980) Strength of polymer and composite materials. Zinatne, Riga

Matveenko VP, Smetannikov OY, Trufanov NA, Shardakov IN (2012) Models of thermomechanical behavior of polymeric materials undergoing glass transition. Acta Mech 223(6):1261-1284

Matveenko VP, Smetannikov OY, Trufanov NA, Shardakov IN (2015) Constitutive relations for viscoelastic materials under thermorelaxation transition. Acta Mech 226(7):2177-2194

Mulina AH, Sawant S (2009) Responses of viscoelastic polymer composites with temperature and time dependent constituents. Acta Mech 204(3):155-173

Nguen TD, Qi HJ, Castro F, Long KN (2008) A thermoviscoelastic model for amorphous shape memory polymers: Incorporating structural and stress relaxation. J Mech Phys Solids 56:2792-2814

Pobedrya BE (1964) Mechanics of composite materials. Moscow University press, Moscow

Qi HJ, Nguen TD, Castro F, Yakacki CM, Shandas R (2008) Finite deformation thermo-mechanical behavior of thermally induced shape memory polymers. J Mech Phys Solids 56(5):1730-1751

Scalet G, Auricchio F, Bonetti E, Castellani L, Ferri D, Pachera M, Scavello F (2015) An experimental, theoretical and numerical investigation of shape memory polymers. Int J Plast 67:127-147

Shardakov IN, Golotina LA (2009) Modeling of the deformation processes in amorphous-crystalline bodies. Comput Continuum Mech 2(3):106-113

Sokolkin Yu V, Tashkinov AA (1984) Mechanics of deformation and fracture of structurally inhomogeneous bodies. Nauka, Moscow

Sollich P (1998) Rheological constitutive equation for a model of soft glassy materials. Phys Rev E 58(1):738-759

Srivastava V, Chester SA, Ames NM, Anand L (2010a) A thermo-mechanicallycoupled large-deformation theory for amorphous polymers in a temperature range which spans their glass transition. Int J Plast 26:1138-1182 
Srivastava V, Chester SA, Anand L (2010b) Thermally actuated shape-memory polymers: experiments, theory and numerical simulations. J Mech Phys Solids 58:1100-1124

Tan Q, Liu L, Liu Y, Leng J (2014) Thermal mechanical constitutive model of fiber reinforced shape memory composite: Based on bridging model. Compos Part A 64:132-138

Tervoort TA, Klompen ETJ, Govaert LE (1996) A multi-mode approach to finite three-dimensional, nonlinear viscoelastic behavior of polymer glasses. J Rheol 40(5):779-797

Vildeman VE, Sokolkin Yu V, Tashkinov AA (1997) Mechanics of inelastic deformation and fracture of composite materials. Nauka, Fismatlit, Moscow

Wang J, Sze KY, Shen YP (2001) Studying the thermomechanical behavior of SM composites with aligned SMA short fibers by micromechanical approaches. Smart Mater Struct 10:990-999

Word I (1975) Mechanical properties of hard polymers. Chemistry, Moscow

Xiao R, Choi J, Lakhera N, Yakacki CM, Frick CP, Nguen TD (2013) Modeling the glass transition of amorphous networks for shape-memory behavior. J Mech Phys Solids 61:1612-1635

\section{Submit your manuscript to a SpringerOpen ${ }^{\odot}$ journal and benefit from:}

- Convenient online submission

- Rigorous peer review

- Immediate publication on acceptance

- Open access: articles freely available online

- High visibility within the field

- Retaining the copyright to your article

Submit your next manuscript at $>$ springeropen.com 